\section{Test, paralleler diagnostischer}

R.-D. Hilgers ${ }^{1}$, N. Heussen ${ }^{1}$ und S. Stanzel ${ }^{2}$

${ }^{1}$ Institut für Medizinische Statistik, Universitätsklinikum der RWTH Aachen, Aachen, Deutschland

${ }^{2}$ DKFZ Heidelberg, Heidelberg, Deutschland

Englischer Begriff parallel diagnostic testing

Definition Unter einem parallelen diagnostischen Test versteht man die gleichzeitige Durchführung mehrerer Einzeltests.

Beschreibung Bei der streng parallelen, also gleichzeitigen Durchführung mehrerer diagnostischer Tests

$(\triangleright$ Test, diagnostischer) gilt die Diagnose üblicherweise schon dann als bestätigt, wenn lediglich einer der Tests positiv ausfällt. Die parallele Durchführung wird meist angewandt, wenn eine schnelle Beurteilung erforderlich ist. Die parallele Durchführung erhöht im Allgemeinen die Sensitivität $(\triangleright$ Sensitivität, diagnostische) und damit den negativen Vorhersagewert ( $\triangleright$ Vorhersagewert, negativer) bei gegebener Prävalenz der Krankheit über die negativen Vorhersagewerte der einzelnen Tests hinaus. Andererseits werden Spezifität $(\triangleright$ Spezifität, diagnostische) und positiver Vorhersagewert ( $\triangleright$ Vorhersagewert, positiver) verringert. Somit ist ein Übersehen der Krankheit weniger wahrscheinlich, aber die Wahrscheinlichkeit für eine falsch-positive Diagnose erhöht.

\section{Literatur}

Hilgers R-D, Bauer P, Scheiber V (2002) Einführung in die Medizinische Statistik. Springer, Berlin/Heidelberg/New York 